This document is the accepted manuscript version of the following article:

Caspari, P., Nüesch, F. A., Neels, A., \& Opris, D. M. (2016). Mild synthesis of mercaptonitriles from vinyl nitriles and their cyclization reactions. RSC Advances, 6(100), 98059-98065. http://doi.org/10.1039/C6RA21948A

\title{
Mild synthesis of mercaptonitriles from vinyl nitriles and their cyclization reactions
}

\author{
Philip Caspari, ${ }^{a, b}$ Frank A. Nüesch, ${ }^{a, b}$ Antonia Neels, ${ }^{c}$ Dorina M. Opris*a
}

\begin{abstract}
Thiol-ene addition of thioacetic acid $\mathbf{A}$ is widely used in the synthesis of thiols from vinyl precursor, but so far was not conducted on non-conjugated vinyl nitriles. The challenge when vinyl nitriles are used is to selectively conduct the thiolene addition, while avoiding the nucleophilic addition of $\mathbf{A}$ to the nitrile group. We have found that vinyl nitriles give selective UV-induced thiol-ene addition in presence of photoinitiators as long as stoichiometric amount of $\mathbf{A}$ to the vinyl group and sterically unhindered vinyls are used. On the contrary, when a sterically hindered vinyl is used, the nucleophilic addition of the nitrile is favoured. The prepared mercaptonitriles can easily undergo cyclization reactions in basic and acidic conditions as well as in presence of silica gel. This illustrates the high reactivity of nitriles towards thiol addition. 1,2ethanedithiol $\mathbf{B}$ is presented as an alternative reagent to $\mathbf{A}$ as it allows conversion of vinyl nitriles directly into mercaptonitriles under mild and non-acidic reaction conditions.
\end{abstract}

\section{Introduction}

The radical addition of thiols to carbon-carbon double bonds is widely known as thiol-ene reaction. This reaction is regioselective and gives the anti-Markovnikov adduct as major product. ${ }^{1}$ Due to high conversion, small amount of by-products and fast reaction time, the thiol-ene reaction is often referred to as "thiol click chemistry" in the literature. ${ }^{2-5}$ The addition is mediated by either thermal ${ }^{6}$ or by UV radical initiators. Thiolene reaction is a versatile tool in biochemistry ${ }^{7-10}$ and polymer science. In polymer science, the thiol-ene reaction is a wellestablished process for the preparation of dithiol monomers ${ }^{6}$ and in the synthesis of polythioethers ${ }^{11-13}$ and polythioesters. ${ }^{14}$ Due to its high conversion it was often used in post-polymerization modifications. ${ }^{15-21}$ When photoinitiators are used, the reaction proceeds to completion within few minutes. ${ }^{7}$ In their absence thiols can be activated by UV irradiation but the reaction rate is considerably lower. ${ }^{8}$ The addition of thioacetic acid $\mathbf{A}$ to alkenes followed by acetyl cleavage is one of the most frequently used synthetic routes to thiols. Cleavage of the acetyl moiety can be conducted under acidic $^{6}$ and basic conditions ${ }^{22}$ or by reduction with lithium aluminium hydride. ${ }^{23}$ Hydrogen sulfide addition to alkenes leads to formation of significant amount of dialkylsulfides sideproducts. Already in 1941 Schjanberg et al. published the selective thiol-ene addition of $\mathbf{A}$ to non-conjugated pentenoic acids. $^{24}$ Later, Brown et al. reported the selective thiol-ene addition of $\mathbf{A}$ to allyl alcohol, allyl acetate, and maleic anhydride mediated by peroxides. ${ }^{25}$ More recently thiol-ene addition of non-conjugated vinyl amides using $\mathrm{BEt}_{3}$ and $\mathrm{O}_{2}$ as initiator was reported. ${ }^{26}$ Thiol-ene addition of aliphatic thiols to allyl cyanide is known, ${ }^{27,28}$ however, the formed sulfides cannot be converted into mercaptonitriles. While selective 1,4addition of $\mathbf{A}$ to acrylonitrile under basic conditions is known, ${ }^{29}$ the thiol-ene addition of $\mathbf{A}$ and 1,2-ethanedithiol $\mathbf{B}$ to nonconjugated vinyl nitrile compounds i.e. allyl cyanide was not explored so far. The addition of $\mathbf{A}$ to aliphatic nitriles was reported to occur under acidic or basic conditions with formation of thioamides. ${ }^{30,31}$ It is also known that mercaptonitriles can undergo intramolecular reactions under acidic conditions. ${ }^{32}$ Given the reactivity of $\mathbf{A}$ to nitriles it was unclear whether a selective thiol-ene addition of $\mathbf{A}$ to various vinyl nitriles followed by the deprotection of the thioester to thiol can be realized.

In polymer science, mercaptonitriles are attractive precursor for the functionalization of polyvinylsiloxanes via thiol-ene reaction. ${ }^{17}$ We have recently shown that the dielectric permittivity $\left(\varepsilon^{\prime}\right)$ of polysiloxanes modified with polar nitrile groups increases linearly with the content of the nitrile groups. By increasing the content of nitrile groups on the polysiloxane, it is to be expected that the permittivity can be further increased. To be attractive precursors for postpolymerization reactions, mercaptonitriles must be synthesized cost-efficiently on g-scale and have to be stable during thiol-ene addition to the polyvinylsiloxane.

This work describes the thiol-ene reaction of $\mathbf{A}$ and 1,2ethanedithiol B to allyl cyanide, allyl malononitrile, and 3-vinyl1,3,5-tricarbopentanitrile which should allow formation of thiols with different content of nitrile groups in two steps or directly, respectively.

\section{Experimental Section}

General Remarks: All reactions were carried out in dried glassware under argon atmosphere. Unless otherwise stated, all chemicals were reagent grade and used as received. Methanol, dichloromethane (DCM), heptane, ethyl acetate, petroleum ether and tetrahydrofurane (THF) were purchased from VWR. Anhydrous methanol was purchased from Sigma Aldrich. Silica gel was purchased from VWR ('Normasil 60' 40-63 $\mu \mathrm{m}$ ). Analytical TLC was carried out on 'TLC Silica gel $60 \mathrm{~F}_{254}$ ' from Merck. TLC plates were visualized with UV light or aqueous $\mathrm{KMnO}_{4}$ solution. THF was dried over sodium and distilled prior use. All other chemicals were 
purchased from Sigma-Aldrich. A mercury vapour UV-light source UVAHAND 250 GSH1 from Dr. Hönle AG without additional filters providing an irradiation intensity of $15 \mathrm{~mW} \mathrm{~cm}^{-2}$ in the frequency range between 320 and $600 \mathrm{~nm}$ was used. ${ }^{1} \mathrm{H}$ and ${ }^{13} \mathrm{C}$ NMR spectra were recorded on a Bruker Avance III 400 NMR spectrometer using a $5 \mathrm{~mm}$ BBO Prodigy ${ }^{\mathrm{TM}}$ CryoProbe at 400.18 and $100.63 \mathrm{MHz}$, respectively. Chemical shifts $(\delta)$ in ppm are calibrated to residual solvent peaks according to literature. ${ }^{33}$ Mass spectrometry was conducted by the laboratory of organic chemistry of ETH Zurich. EIMS was measured with Waters' AutoSpec Ultima (El-triSector-MS), ESI-MS with Bruker's maXis (ESI/NanoSpray-Qq-TOF-MS). Elemental analysis was determined with LECO TruSpec Micro, LECO RO-478 and LECO CHNS-932 by the laboratory of organic chemistry of ETH Zurich. For the broadband dielectric spectroscopy (BDS) measurements a high impedance Alpha Analyzer combined with a Quatro temperature controller (both from Novocontrol) has been employed to cover a broad frequency from $0.1 \mathrm{~Hz}$ to $1 \mathrm{MHz}$. Two stainless steel discs with a diameter of $20 \mathrm{~mm}$ served as electrodes which were separated by three glass fibers with a diameter of 100 $\mu \mathrm{m}$.

S-3-cyanopropyl thioacetate (1): Allyl cyanide $(20.0 \mathrm{ml}, 0.25 \mathrm{~mol})$, thioacetic acid $(26.6 \mathrm{ml}, 0.38 \mathrm{~mol})$, and 2,2-dimethoxy-2phenylacetophenone (DMPA) $(0.64 \mathrm{~g}, 0.0025 \mathrm{~mol})$ were dissolved in THF. The solution was degassed and irradiated for 5 min with a UV light. All volatiles were removed in vacuo at $60{ }^{\circ} \mathrm{C}$ to obtain $36 \mathrm{~g}$ crude product mixture. The residue was fractionally distilled at 117 ${ }^{\circ} \mathrm{C}$ and $1 \times 10^{-3} \mathrm{mbar}$ to give $1(25.4 \mathrm{~g}, 73 \%)$ as pale yellowish liquid. ${ }^{1} \mathrm{H}$ NMR $\left(400 \mathrm{MHz}, \mathrm{CDCl}_{3}\right) \delta$, ppm: $2.97\left(\mathrm{t},{ }^{3} \mathrm{~J}=6.9 \mathrm{~Hz}, 2 \mathrm{H},-\mathrm{S}-\mathrm{C}_{2}-\mathrm{CH}_{2}-\right.$ ), $2.42\left(\mathrm{t},{ }^{3} \mathrm{~J}=8.0 \mathrm{~Hz}, 2 \mathrm{H},-\mathrm{CH}_{2}-\mathrm{CH}_{2}-\mathrm{CN}\right.$ ), 2.35 (s, 3H, $\left.\underline{\mathrm{CH}}_{3}-\mathrm{CO}-\mathrm{S}-\right), 1.94$ (q, $\left.{ }^{3}=7.1 \mathrm{~Hz}, 2 \mathrm{H},-\mathrm{CH}_{2}-\mathrm{CH}_{2}-\mathrm{CH}_{2}-\mathrm{CN}\right) .{ }^{13} \mathrm{C} \mathrm{NMR}\left(100 \mathrm{MHz}, \mathrm{CDCl}_{3}\right) \delta$, ppm: 195.33 ( $\left.\mathrm{CH}_{3}-\underline{\mathrm{CO}}-\mathrm{S}-\right), 119.09$ (- $\left.\mathrm{CH}_{2}-\mathrm{CH}_{2}-\underline{\mathrm{CN}}\right), 30.77\left(\underline{\mathrm{C}} \mathrm{H}_{3}-\mathrm{CO}-\mathrm{S}-\right)$, 28.00 (-S- $\left.\mathrm{CH}_{2}-\mathrm{CH}_{2}-\right), 25.93\left(-\mathrm{S}-\mathrm{CH}_{2}-\underline{C H}_{2}-\right), 16.42\left(-\mathrm{CH}_{2}-\underline{C H}_{2}-\mathrm{CN}\right) . \mathrm{MS}$ (EI) $m / z$ (\%): 43.02 (100), 128.02 (10.97), 143.04 (4.38). EA: calculated: [C] 50.32\% [H] 6.33\% [N] 9.78\% [O] 11.17 [S] 22.39 found: [C] 50.27\% [H] 6.54\% [N] 9.54\% [O] 11.40 [S] 22.22\%.

4-Mercaptobutanenitrile (2): 4-thioacetic butanenitrile 1 (10 g, 0.07 mol), trimethylsilyl chloride $(1.8 \mathrm{ml}, 0.014 \mathrm{~mol})$, and methanol (150 $\mathrm{ml}, 52 \mathrm{~mol}$ ) were stirred at $65^{\circ} \mathrm{C}$ for $18 \mathrm{~h}$. The reaction mixture was allowed to cool down to ambient temperature and all volatiles were removed in vacuo. The residue was dissolved in chloroform and filtered. After removing all volatiles in vacuo, $4.3 \mathrm{~g}$ of crude product was obtained. The residue was fractionally distilled at $77^{\circ} \mathrm{C}$ and 10 mbar to give $2(3 \mathrm{~g}, 43 \%)$ as colourless liquid. ${ }^{1} \mathrm{H}$ NMR $(400 \mathrm{MHz}$, $\left.\mathrm{CDCl}_{3}\right) \delta$, ppm: $2.68\left(\mathrm{dt},{ }^{3} J=7.0 \mathrm{~Hz}, 8.3 \mathrm{~Hz}, 2 \mathrm{H}, \mathrm{HS}-\mathrm{CH}_{2}-\mathrm{CH}_{2}-\right), 2.54(\mathrm{t}$, ${ }^{3} \mathrm{~J}=7.0 \mathrm{~Hz}, 2 \mathrm{H},-\mathrm{CH}_{2}-\mathrm{CH}_{2}-\mathrm{CN}$ ), 1.96 (q, ${ }^{3} \mathrm{~J}=7.0 \mathrm{~Hz}, 2 \mathrm{H},-\mathrm{CH}_{2}-\mathrm{CH}_{2}-\mathrm{CH}_{2}-$ ), $1.38\left(\mathrm{t},{ }^{3} \mathrm{~J}=8.3 \mathrm{~Hz}, 2 \mathrm{H}, \underline{\mathrm{HS}}-\mathrm{CH}_{2}-\right) .{ }^{13} \mathrm{C}$ NMR $\left(100 \mathrm{MHz}, \mathrm{CDCl}_{3}\right) \delta, \mathrm{ppm}$ : $119.10\left(-\mathrm{CH}_{2}-\mathrm{CH}_{2}-\underline{\mathrm{CN}}\right), 29.39\left(-\mathrm{S}-\underline{C H}_{2}-\mathrm{CH}_{2}-\right), 23.34$ (-S- $\left.-\mathrm{CH}_{2}-\underline{C H}_{2}-\right)$, 15.90 (- $\mathrm{CH}_{2}-\mathrm{CH}_{2}-\mathrm{CN}$ ). MS (EI) $m / z$ (\%): 54.03 (100), 68.05 (23.84), 101.03 (97.52).

2-Iminothiolane hydrochloride (3): 1 (1.4 g, $0.01 \mathrm{~mol}$ ), trimethylsilyl chloride $(1.3 \mathrm{ml}, 0.01 \mathrm{~mol})$, and methanol $(20.2 \mathrm{ml}, 52 \mathrm{~mol})$ were stirred at $65{ }^{\circ} \mathrm{C}$ for $18 \mathrm{~h}$. The reaction mixture was allowed to cool down to ambient temperature and all volatiles were removed in vacuo. The residue was washed with chloroform to give $\mathbf{3}(0.6 \mathrm{~g}$, $45 \%)$ as fine powder. ${ }^{1} \mathrm{H} \mathrm{NMR}\left(400 \mathrm{MHz}, \mathrm{DMSO}_{-} \mathrm{d}_{6}\right) \delta$, ppm: 12.37 (s, $\left.2 \mathrm{H},-\mathrm{CH}_{2}=\mathrm{NH}_{2}\right), 3.51\left(\mathrm{t},{ }^{3} \mathrm{~J}=6.6 \mathrm{~Hz}, 2 \mathrm{H},-\mathrm{CH}_{2}-\mathrm{CH}_{2}-\mathrm{S}-\right), 3.18\left(\mathrm{t},{ }^{3} \mathrm{~J}=7.1 \mathrm{~Hz}\right.$, $\left.2 \mathrm{H},-\mathrm{C}\left(=\mathrm{NH}_{2}\right)-\mathrm{CH}_{2}-\mathrm{CH}_{2}-\right), 2.56\left(\mathrm{q},{ }^{3} \mathrm{~J}=6.8 \mathrm{~Hz}, 2 \mathrm{H},-\mathrm{CH}_{2}-\mathrm{CH}_{2}-\mathrm{CH}_{2}-\right) .{ }^{13} \mathrm{C}$ NMR (100 MHz, DMSO-d $\left.\mathrm{d}_{6}\right) \delta$, ppm: $203.78\left(-\mathrm{S}-\underline{\mathrm{C}}=\mathrm{NH}_{2}\right), 39.38$ (-S$\left.\underline{\mathrm{C}} \mathrm{H}_{2}-\mathrm{CH}_{2}-\right), 37.61\left(-\mathrm{C}\left(=\mathrm{NH}_{2}\right)-\underline{-} \mathrm{CH}_{2}-\mathrm{CH}_{2}-\right), 27.55\left(-\mathrm{CH}_{2}-\underline{C H}_{2}-\mathrm{CH}_{2}-\right)$.

Allylmalononitrile (5): To a solution of malononitrile $(66 \mathrm{~g}, 1 \mathrm{~mol})$ in DCM $(300 \mathrm{ml})$, allyl bromide $(86 \mathrm{ml}, 1 \mathrm{~mol})$ and subsequently diisopropylethylamine $(165 \mathrm{ml}, 0.95 \mathrm{~mol})$ were dropped slowly at 0 ${ }^{\circ} \mathrm{C}$. The solution was allowed to warm up and stirred overnight. The reaction mixture was washed several times with $\mathrm{H}_{2} \mathrm{O}$. The organic phase was separated and dried over $\mathrm{MgSO}_{4}$, filtered and concentrated in vacuo to give $83 \mathrm{~g}$ crude product mixture. It was purified by column chromatography (petroleum ether $\left(40-60{ }^{\circ} \mathrm{C}\right.$ )/ ethyl acetate 9:1) to give $\mathbf{5}$ ( $35 \mathrm{~g}, 35 \%)$ as colourless viscous liquid. $\mathrm{R}_{\mathrm{F}}=0.4$ (heptane/ethyl acetate 2:1). ${ }^{1} \mathrm{H}$ NMR $\left(400 \mathrm{MHz}, \mathrm{CDCl}_{3}\right) \delta$, ppm: 5.91-5.80 (m, $\left.1 \mathrm{H},-\mathrm{CH}_{2}-\underline{\mathrm{C}}=\mathrm{CH}_{2}\right), 5.43-5.38\left(\mathrm{~m}, 2 \mathrm{H},-\mathrm{CH}_{2}-\right.$ $\left.\mathrm{CH}=\mathrm{CH}_{2}\right), 3.78\left(\mathrm{t},{ }^{3} \mathrm{~J}=6 \mathrm{~Hz}, 1 \mathrm{H},(\mathrm{NC})_{2}-\mathrm{CH}_{-}-\mathrm{CH}_{2}-,\right), 2.75\left(\mathrm{dt},{ }^{4} \mathrm{~J}=1 \mathrm{~Hz}\right.$, $\left.{ }^{3} \mathrm{~J}=6.6 \mathrm{~Hz} 2 \mathrm{H}, \mathrm{CH}-\mathrm{CH}_{2}-\mathrm{CH}=\right) .{ }^{13} \mathrm{C} \mathrm{NMR}\left(100 \mathrm{MHz}, \mathrm{CDCl}_{3}\right) \delta, \mathrm{ppm}$ : $129.29\left(-\underline{\mathrm{C}} \mathrm{H}=\mathrm{CH}_{2}\right), 122.55\left(-\mathrm{CH}=\underline{\mathrm{C}} \mathrm{H}_{2}\right), 112.35(-\mathrm{CN}), 34.74\left(-\mathrm{CH}-\underline{\mathrm{C}} \mathrm{H}_{2}-\right)$, $23.16\left(-\underline{C} \mathrm{H}-\mathrm{CH}_{2}-\right)$.

S-(4,4-dicyanobutyl) thioacetate (6): A solution of allyl malononitrile $(1.06 \mathrm{~g}, 0.01 \mathrm{~mol})$, thioacetic acid $(1.2 \mathrm{ml}, 0.012 \mathrm{~mol})$ and DMPA $(0.025 \mathrm{~g}, 0.0002 \mathrm{~mol})$ in THF $(15 \mathrm{ml})$ was carefully degassed and then irradiated for $5 \mathrm{~min}$ with UV light. The volatiles were removed in vacuo and the crude product mixture $(2.6 \mathrm{~g})$ was purified by column chromatography (Heptane/Ethyl acetate 2:1, $R_{F}=$ 0.3). 6 was obtained as colourless viscous liquid (1.35 g, 74\%). ${ }^{1} \mathrm{H}$ NMR (400 MHz, CDCl ${ }_{3}$ ) $\delta$, ppm: $3.87\left(\mathrm{t},{ }^{3} J=6.8 \mathrm{~Hz}, 1 \mathrm{H},(\mathrm{NC})_{2}-\mathrm{C}_{-}-\mathrm{CH}_{2}-\right.$ ), $2.95\left(\mathrm{t},{ }^{3} \mathrm{~J}=6.8 \mathrm{~Hz}, 2 \mathrm{H}, \mathrm{CH}_{2}-\mathrm{CH}_{2}-\mathrm{SAc}\right), 2.36\left(\mathrm{~s}, 3 \mathrm{H}, \mathrm{CH}_{2}-\mathrm{S}-\mathrm{CH}_{3}\right), 2.13-$ $2.06\left(\mathrm{~m}, 2 \mathrm{H}, \mathrm{CH}-\mathrm{CH}_{2}-\mathrm{CH}_{2}-\right), 1.95-1.84\left(\mathrm{~m}, 2 \mathrm{H}, \mathrm{CH}-\mathrm{CH}_{2}-\mathrm{CH}_{2}-\right) .{ }^{13} \mathrm{C} \mathrm{NMR}$ $\left(100 \mathrm{MHz}, \mathrm{CDCl}_{3}\right) \delta$, ppm: $195.65\left(-\mathrm{S}-\underline{\mathrm{C}}=\mathrm{O}-\mathrm{CH}_{3}\right), 112.53(-\underline{\mathrm{CN}}), 30.76$ $\left(-\mathrm{S}-\mathrm{C}(=\mathrm{O})-\underline{-} \mathrm{CH}_{3}\right), 29.55\left(\mathrm{CH}_{2}-\underline{\mathrm{C}} \mathrm{H}_{2}-\mathrm{CH}_{2}\right), 27.22\left(\underline{\mathrm{C}} \mathrm{H}_{2}-\mathrm{SAC}\right), 26.84(\mathrm{CH}-$ $\left.\underline{\mathrm{C}}_{2}-\right), 22.30\left(-\underline{\mathrm{C}} \mathrm{H}-\mathrm{CH}_{2}-\right) . \mathrm{MS}(\mathrm{El}+) \mathrm{m} / \mathrm{z}(\%): 182.05$ (5.53), 43.02 (100). EA: calculated: [C] $52.73 \%$ [H] 5.53\% [N] 15.37\% [O] 8.78\% [S] $17.59 \%$ found: [C] $52.53 \%$ [H] 5.75\% [N] 15.31\% [O] 9.05 [S] 17.53\%.

4,4-dicyanobutyl thiol (7): To a degassed solution of 6 (4.7 g, 0.025 mol) in anhydrous $\mathrm{MeOH}(43 \mathrm{ml}, 1.07 \mathrm{~mol}$ ) trimethylsilyl chloride was added $(0.54 \mathrm{ml}, 0.0043 \mathrm{~mol})$. The reaction was refluxed for 18 h. The reaction was allowed to cool down to ambient temperature. All volatiles were removed in vacuo. The residue was redissolved in $\mathrm{CHCl}_{3}$ and filtered through a syringe filter. After removing all volatiles in vacuo $3.5 \mathrm{~g}$ yellowish viscous liquid was obtained. The residue was fractionally distilled to give $2.5 \mathrm{~g}$ pale yellowish liquid (71\%; purity 85\%). Further attempts to purify this compound by column chromatography were not successful. 7 gives a cyclization reaction to 8 during this purification step. ${ }^{1} \mathrm{H}$ NMR ( $400 \mathrm{MHz}, \mathrm{CDCl}_{3}$ ) $\delta$, ppm: $3.80\left(\mathrm{t},{ }^{3} \mathrm{~J}=6.8 \mathrm{~Hz}, 1 \mathrm{H},(\mathrm{NC})_{2}-\mathrm{CH}_{-}-\mathrm{CH}_{2}-\right), 2.61-2.67(\mathrm{~m}, 2 \mathrm{H}$, $\left.\mathrm{CH}_{2}-\mathrm{CH}_{2}-\mathrm{SH}\right), 2.23-2.17\left(\mathrm{~m}, 2 \mathrm{H}, \mathrm{CH}-\mathrm{CH}_{2}-\mathrm{CH}_{2}-\right), 1.98-1.90(\mathrm{~m}, 2 \mathrm{H}, \mathrm{CH}-$ $\left.\mathrm{CH}_{2}-\underline{\mathrm{C}}_{2}-\right), 1.43$ (t, $\left.{ }^{3}=8.1 \mathrm{~Hz}, 1 \mathrm{H},-\mathrm{CH}_{2}-\mathrm{S} \underline{\mathrm{H}}\right) .{ }^{13} \mathrm{C} \mathrm{NMR}(100 \mathrm{MHz}$, $\left.\mathrm{CDCl}_{3}\right) \delta$, ppm: 195.60 (-S- $\left.\underline{\mathrm{C}}=\mathrm{O}-\mathrm{CH}_{3}\right), 112.54(-\underline{\mathrm{CN}}), 29.47\left(\mathrm{CH}_{2}-\underline{\mathrm{CH}}_{2}-\right.$ $\left.\mathrm{CH}_{2}\right), 30.39\left(\underline{\mathrm{CH}}_{2}-\mathrm{SH}\right), 23.39\left(\mathrm{CH}-\mathrm{CH}_{2}-\right), 22.46\left(-\mathrm{CH}-\underline{\mathrm{C}} \mathrm{H}_{2}-\right)$. HRMS (ESI): calc. $\mathrm{C}_{6} \mathrm{H}_{9} \mathrm{~N}_{2} \mathrm{~S} \mathrm{~m} / \mathrm{z}$ : $141.0481[\mathrm{M}+\mathrm{H}]$; found $141.0481[\mathrm{M}+\mathrm{H}]$.

6-Amino-3,4-dihydro-2H-thiopyran-5-carbonitrile (8): To a degassed solution of 6 (2.6 g, $0.014 \mathrm{~mol})$ in anhydrous $\mathrm{MeOH}(24$ $\mathrm{ml}, 0.593 \mathrm{~mol})$ trimethylsilyl chloride was added $(0.03 \mathrm{ml}, 0.0024$ 
mol). The reaction was refluxed for $17 \mathrm{~h}$. Then, additional trimethylsilyl chloride $(0.03 \mathrm{ml})$ was added and the reaction mixture stirred for another $72 \mathrm{~h}$ at r.t. All volatiles were removed in vacuo. The residue was redissolved in $\mathrm{CHCl}_{3}$ and filtered through a syringe filter. After removing all volatiles in vacuo $1.63 \mathrm{~g}$ yellow viscous liquid was obtained. An aliquot of $0.5 \mathrm{~g}$ of crude product mixture was purified by column chromatography and recrystallized from $n$ heptane/ ethyl acetate to give $8(0.3 \mathrm{~g}, 49 \%)$ as colourless solid. $R_{F}=$ 0.2 (DCM/ heptane 3:1). ${ }^{1} \mathrm{H}$ NMR $\left(400 \mathrm{MHz}, \mathrm{CDCl}_{3}\right) \delta$, ppm: $4.33(\mathrm{br}$ $\left.\mathrm{s}, 2 \mathrm{H},-\mathrm{NH}_{2}\right), 2.94\left(\mathrm{t},{ }^{3} \mathrm{~J}=5.6 \mathrm{~Hz}, 2 \mathrm{H},-\mathrm{CH}_{2}-\mathrm{CH}_{2}-\mathrm{S}\right), 2.35\left(\mathrm{t},{ }^{3} \mathrm{~J}=6.4 \mathrm{~Hz}\right.$, $\left.2 \mathrm{H},-\mathrm{CH}_{2}-\mathrm{CH}_{2}-\mathrm{CH}_{2}-\right)$, 2.03-1.96 (m, 2H, $\left.-\mathrm{CH}_{2}-\mathrm{CH}_{2}-\mathrm{CH}_{2}\right){ }^{13} \mathrm{C}$ NMR (100 $\left.\mathrm{MHz}, \mathrm{CDCl}_{3}\right) \delta$, ppm: $154.19\left(\mathrm{C}=\underline{\mathrm{C}}\left(-\mathrm{NH}_{2}\right)-\mathrm{S}\right), 120.13(-\underline{\mathrm{CN}}), 72.94$ ((NC)- $\underline{C}=\mathrm{C}-\mathrm{S}), 28.17\left(\mathrm{~S}-\underline{\mathrm{C}} \mathrm{H}_{2}-\mathrm{CH}_{2}-\mathrm{CH}_{2}-\right), 24.37\left(\mathrm{~S}-\mathrm{CH}_{2}-\mathrm{CH}_{2}-\underline{\mathrm{CH}_{2}}-\right), 22.47$ $\left(-\mathrm{CH}_{2}-\mathrm{CH}_{2}-\mathrm{CH}_{2}-\right)$. HRMS (ESI) calcd. for $\mathrm{C}_{6} \mathrm{H}_{9} \mathrm{~N}_{2} \mathrm{~S}[\mathrm{M}+\mathrm{H}]$ 141.0481, found 141.0481. EA: calculated: [C] 51.40\% [H] 5.74\% [N] 19.98\% found: [C] 51.44\% [H] 5.83\% [N] 19.73\%.

3-Vinylpentane-1,3,5-trinitrile (9): Allyl cyanide (12.1 ml, $0.15 \mathrm{~mol})$ and vinyl cyanide $(21 \mathrm{ml}, 0.32 \mathrm{~mol})$ were added dropwise to a mixture of trimethylbenzylammonium hydroxide $(1.2 \mathrm{ml}$ of $40 \mathrm{wt} \%$ aqueous solution, $0.003 \mathrm{~mol})$ in tert-butanol $(25 \mathrm{ml})$ at $25^{\circ} \mathrm{C}$. The temperature was kept constant by use of an ice bath. After complete addition, the reaction mixture was stirred overnight at ambient temperature. The mixture was acidified with diluted aqueous $\mathrm{HCl}$ and washed several times with DCM. The organic phase was dried over $\mathrm{MgSO}_{4}$ and the volatiles were removed in vacuo. The residue was purified by column chromatography ( $n$ heptane/ethyl acetate $2: 1$ ) to give a pale yellowish solid $(9.0 \mathrm{~g})$. The solid was dissolved in ethyl acetate and precipitated by slow addition $n$-heptane to obtain $9(8.2 \mathrm{~g}, 31 \%)$ as colourless solid. $R_{F}=$ 0.5 (heptane/ ethyl acetate 1:1). ${ }^{1} \mathrm{H}$ NMR $\left(400 \mathrm{MHz}, \mathrm{CDCl}_{3}\right) \delta$, ppm: $5.67\left(\mathrm{~d},{ }^{3} \mathrm{~J}=16 \mathrm{~Hz}, 1 \mathrm{H}, \mathrm{CH}\right.$ 5.44 (dd, ${ }^{3} \mathrm{~J}=10.2 \mathrm{~Hz},{ }^{3} \mathrm{~J}=16.8 \mathrm{~Hz}, 1 \mathrm{H}, \mathrm{CH}_{2}=\mathrm{C} \underline{\mathrm{H}}-$ ) , 2.57-2.39 (m, $4 \mathrm{H}$, $\left.\mathrm{CH}_{2}-\mathrm{CH}_{2}-\mathrm{CN}\right), 2.24-2.17\left(\mathrm{~m}, 2 \mathrm{H}, \mathrm{CH} \underline{H}-\mathrm{CH}_{2}-\mathrm{CN}\right), 2.00-1.92(\mathrm{~m}, 2 \mathrm{H}$, $\left.\mathrm{CH} \underline{H}-\mathrm{CH}_{2}-\mathrm{CN}\right) .{ }^{13} \mathrm{C}$ NMR $\left(100 \mathrm{MHz}, \mathrm{CDCl}_{3}\right) \delta, \mathrm{ppm}: 132.76\left(\mathrm{CH}_{2}=\underline{\mathrm{CH}}-\right)$, $122.17\left(\underline{\mathrm{C}} \mathrm{H}_{2}=\mathrm{CH}-\right), 117.98\left(\mathrm{C}_{\text {quart }}-\underline{\mathrm{CN}}\right), 117.85\left(\mathrm{CH}_{2}-\underline{\mathrm{CN}}\right), 45.81\left(\mathrm{C}_{\text {quart }}\right)$, $34.22\left(\underline{C}_{2}-\mathrm{CH}_{2} \mathrm{CN}\right), 13.66\left(\mathrm{CH}_{2}-\underline{C H}_{2}-\mathrm{CN}\right)$. MS (EI) $\mathrm{m} / \mathrm{z}$ (\%): 55.05 (49.53), 80.05 (34.74), 92.05 (70.69), 119.06 (100). EA: calculated: [C] $69.34 \%[\mathrm{H}] 6.40 \%[\mathrm{~N}] 24.26 \%$ found: [C] 69.21\% [H] 6.33\% [N] $24.16 \%$.

4-Cyano-4-vinylheptane bis(thioamide) (12): A solution of 3vinylpentane-1,3,5-tricarbonitrile $(0.5 \mathrm{~g}, 0.0029 \mathrm{~mol})$, thioacetic acid $(4.8 \mathrm{ml}, 0.024 \mathrm{~mol})$, and AIBN $(0.015 \mathrm{~g}, 0.09 \mathrm{mmol})$ dissolved in toluene $(30 \mathrm{ml})$ was carefully degassed. The reaction mixture was stirred for $18 \mathrm{~h}$ at $70{ }^{\circ} \mathrm{C}$. After removing all volatiles in vacuo, the mixture was purified by column chromatography ( $n$-heptane/ethyl acetate $1: 1)$ to give $(0.4 \mathrm{~g}, 58 \%)$ of $\mathbf{1 2} . \mathrm{R}_{\mathrm{F}}=0.2$ (heptane/ethyl acetate $1: 1) .{ }^{1} \mathrm{H}$ NMR (400 MHz, acetone- $\left.\mathrm{d}_{6}\right) \delta, \mathrm{ppm}: 8.68$ (br s, $4 \mathrm{H}$, CS-N $\underline{H}_{2}$ ), 5.71 (dd, $\left.{ }^{3} \mathrm{~J}=10 \mathrm{~Hz},{ }^{3} \mathrm{~J}=17 \mathrm{~Hz}, 1 \mathrm{H}, \mathrm{CH}_{2}=\mathrm{CH}-\right), 5.42\left(\mathrm{~d},{ }^{3} \mathrm{~J}=17\right.$ $\mathrm{Hz}, 1 \mathrm{H}, \mathrm{CH} \underline{H}=\mathrm{CH}-), 5.35\left(\mathrm{~d},{ }^{3} \mathrm{~J}=10 \mathrm{~Hz}, 1 \mathrm{H}, \underline{\mathrm{C}} \underline{\mathrm{H}}=\mathrm{CH}-\right), 2.77-2.63(\mathrm{~m}$, $\left.4 \mathrm{H}, \mathrm{CH}_{2}-\mathrm{CH}_{2}-\mathrm{C}(\mathrm{S}) \mathrm{NH}_{2}\right), 2.28-2.11\left(\mathrm{~m}, 4 \mathrm{H}, \mathrm{CH}_{2}-\mathrm{CH}_{2}-\mathrm{CN}\right) .{ }^{13} \mathrm{C}$ NMR (100 $\mathrm{MHz}$, acetone-d6) $\delta$, ppm: $208.12\left(-\underline{\mathrm{C}}(\mathrm{S}) \mathrm{NH}_{2}\right), 136.79\left(\mathrm{CH}_{2}=\underline{\mathrm{C}} \mathrm{H}-\right)$, $120.21\left(\underline{\mathrm{C}} \mathrm{H}_{2}=\mathrm{CH}-\right), 117.37\left(\mathrm{C}_{\text {quart }}-\underline{C N}\right), 45.60\left(\mathrm{C}_{\text {quart }}\right), 40.14\left(\underline{\mathrm{C}}_{2}-\right.$ $\left.\underline{\mathrm{C}} \mathrm{H}_{2} \mathrm{C}(\mathrm{S}) \mathrm{NH}_{2}\right)$, $37.31\left(\mathrm{CH}_{2}-\underline{C H}_{2}-\mathrm{C}(\mathrm{S}) \mathrm{NH}_{2}\right)$; $\mathrm{MS}$ (ESI): calc. for $\mathrm{C}_{10} \mathrm{H}_{16} \mathrm{~N}_{3} \mathrm{~S}_{2}$ $[\mathrm{M}+\mathrm{H}]$ 242.0780; found 242.0779 .
4-((2-Mercaptoethyl)thio)butanenitrile (13): Allyl cyanide $(20 \mathrm{ml}$, $0.25 \mathrm{~mol}), 1,2$-ethanedithiol (freshly distilled) $(168 \mathrm{ml}, 2 \mathrm{~mol})$, and DMPA (1.3 g, $0.005 \mathrm{~mol})$ were solved in THF. The solution was degassed and irradiated for $5 \mathrm{~min}$. All volatiles were removed in vacuo at $60^{\circ} \mathrm{C}$ to obtain $46 \mathrm{~g}$ crude product mixture. The residue was fractionally distilled at $105^{\circ} \mathrm{C}$ and $1 \times 10^{-3}$ mbar to give 13 (26.2 g, 67\%) as colourless liquid (95\% Anti-Markovnikov product). ${ }^{1} \mathrm{H}$ NMR $\left(400 \mathrm{MHz}, \mathrm{CDCl}_{3}\right) \delta$, ppm: 2.76-2.71 (m, 4H, $\left.-\mathrm{S}-\mathrm{C}_{2}-\mathrm{CH}_{2}-\mathrm{SH}\right)$, $2.68\left(\mathrm{t},{ }^{3} \mathrm{~J}=7.2 \mathrm{~Hz}, 2 \mathrm{H},-\mathrm{S}-\mathrm{CH}_{2}-\mathrm{CH}_{2}-\right), 2.51\left(\mathrm{t}, 2 \mathrm{H},-\mathrm{CH}_{2}-\mathrm{CH}_{2}-\mathrm{CN}, \mathrm{J}=6.8\right.$ $\mathrm{Hz}), 1.94\left(\mathrm{dt},{ }^{4} \mathrm{~J}=7.44 \mathrm{~Hz}, 2 \mathrm{H},-\mathrm{CH}_{2}-\mathrm{CH}_{2}-\mathrm{CH}_{2}-\mathrm{CN}\right), 1.71\left(\mathrm{t},{ }^{3} \mathrm{~J}=8.1 \mathrm{~Hz}\right.$, $\left.1 \mathrm{H},-\mathrm{CH}_{2}-\mathrm{S} \underline{\mathrm{H}}\right){ }^{13}{ }^{13} \mathrm{CNMR}\left(100 \mathrm{MHz}, \mathrm{CDCl}_{3}\right) \delta$, ppm: $119.27\left(\mathrm{CH}_{2}-\underline{\mathrm{CN}}\right)$, $36.20\left(\mathrm{HS}-\mathrm{CH}_{2}-\underline{\mathrm{C}} \mathrm{H}_{2}-\mathrm{S}-\right)$, $30.66\left(-\mathrm{S}-\mathrm{CH}_{2}-\mathrm{CH}_{2}-\right), 25.36\left(-\mathrm{CH}_{2}-\underline{C H}_{2}-\mathrm{CH}_{2}-\right)$, $24.77\left(\mathrm{HS}-\mathrm{CH}_{2}-\mathrm{CH}_{2}-\right), 16.14\left(-\mathrm{CH}_{2}-\mathrm{CH}_{2}-\mathrm{CN}\right) . \mathrm{MS}[\mathrm{El}+] \mathrm{m} / \mathrm{z}$ (\%): 114.03 (94.2), 161.03 (60.5). EA: calculated: [C] 44.68\% [H] 6.87\% [N] 8.68\% [S] 39.76 found: [C] 44.51\% [H] 7.12\% [N] 8.58\% [S] 39.78.

2-(3-((2-Mercaptoethyl)thio)propyl)malononitrile (14): To a solution of allyl malononitrile $(15 \mathrm{~g}, 0.14 \mathrm{~mol})$ dissolved in freshly distilled THF (500 $\mathrm{ml})$, freshly distilled 1,2-ethanedithiol $(52 \mathrm{ml}, 0.70$ mol) and DMPA (0.7 g, $0.003 \mathrm{~mol})$ was added under argon atmosphere. The reaction mixture was irradiated for 5 minutes. All volatiles were removed in vacuo (10 mbar). The residue was fractionally distilled at $145{ }^{\circ} \mathrm{C}$ and $9 \times 10^{-3}$ mbar to give 14 as colourless liquid $(9 \mathrm{~g}, 31 \%) .{ }^{1} \mathrm{H}$ NMR $\left(400 \mathrm{MHz}, \mathrm{CDCl}_{3}\right) \delta$, ppm: 3.83 $\left(\mathrm{t},{ }^{3} \mathrm{~J}=6.8 \mathrm{~Hz}, 1 \mathrm{H},(\mathrm{CN}) \mathrm{CH}_{-}-\mathrm{CH}_{2}-\right), 2.79-2.69\left(\mathrm{~m}, 4 \mathrm{H},-\mathrm{S}-\left(\mathrm{CH}_{2}\right)_{2}-\mathrm{SH}\right), 2.64$ $\left(\mathrm{t},{ }^{3} \mathrm{~J}=7.1 \mathrm{~Hz}, 2 \mathrm{H}, \mathrm{CH}_{2}-\mathrm{CH}_{2}-\mathrm{S}-,\right), 2.21-2.15\left(\mathrm{~m}, 2 \mathrm{H}, \mathrm{CH}-\mathrm{CH}_{2}-\mathrm{CH}_{2}\right), 1.96-$ $1.88\left(\mathrm{~m}, 2 \mathrm{H}, \mathrm{CH}_{2}-\mathrm{CH}_{2}-\mathrm{CH}_{2}\right), 1.72\left(\mathrm{t},{ }^{3} \mathrm{~J}=8.0 \mathrm{~Hz}, 1 \mathrm{H}, \mathrm{CH}_{2}-\mathrm{S}-\underline{H},\right) .{ }^{13} \mathrm{C} \mathrm{NMR}$ (100 MHz, CDCl 3$) \delta$, ppm: $112.47\left((\underline{\mathrm{CN}})_{2}-\mathrm{CH}-\right), 36.11\left(-\underline{\mathrm{CH}}_{2}-\mathrm{CH}_{2}-\mathrm{SH}\right)$, $30.62\left(-\underline{\mathrm{C}} \mathrm{H}_{2}-\mathrm{CH}_{2}-\mathrm{S}-\right), 29.64\left(\mathrm{CH}-\underline{\mathrm{CH}} \mathrm{H}_{2}-\mathrm{CH}_{2}\right), 26.11\left(\mathrm{CH}_{2}-\underline{\mathrm{CH}}_{2}-\mathrm{CH}_{2}\right), 24.72$ $\left(\mathrm{CH}_{2}-\mathrm{CH}_{2}-\mathrm{SH}\right), 22.48$ ((CN) $\left.{ }_{2}-\underline{\mathrm{C}} \mathrm{H}-\right)$. MS (ESI) m/z (\%): 167 (100), 359.24 (22.5), 423 (23.8). EA: calculated: [C] 47.97\% [H] 6.04\% [N] 13.98\% [S] 32.01\%/found: [C] 47.90\% [H] 6.24\% [N] 13.74\% [S] 31.86\%.

\section{3-(2-((2-Mercaptoethyl)thio)ethyl)pentane-1,3,5-tricarbonitrile}

(15): A solution of $9(0.173 \mathrm{~g}, 0.001 \mathrm{~mol})$, freshly distilled 1,2ethanedithiol $(0.84 \mathrm{ml}, 0.01 \mathrm{~mol})$, and DMPA $(0.77 \mathrm{~g}, 0.003 \mathrm{~mol})$ in THF $(20 \mathrm{ml})$ was carefully degassed and then irradiated for $5 \mathrm{~min}$ with a UV lamp. To the reaction mixture additional 1,2ethanedithiol $(0.42 \mathrm{ml}, 0.004 \mathrm{~mol})$ and DMPA ( $0.77 \mathrm{~g}, 0.003 \mathrm{~mol})$ were added followed by $10 \mathrm{~min}$ irradiation. All volatiles were removed in vacuo and the product was purified by column chromatography (Heptane/ Ethyl acetate $1: 1)$ to give $(0.09 \mathrm{~g}, 34 \%)$ 15 as yellowish liquid. ${ }^{1} \mathrm{H} \mathrm{NMR}\left(400 \mathrm{MHz}, \mathrm{CDCl}_{3}\right) \delta$, ppm: $2.83-2.72$ (m, $\left.4 \mathrm{H}, \mathrm{HS}-\mathrm{C}_{2}-\mathrm{C}_{2}-\mathrm{S}\right), 2.67$ (t, $2 \mathrm{H},-\mathrm{S}-\mathrm{C}_{2}-\mathrm{CH}_{2}-\mathrm{C}_{\text {quart }}{ }^{3} \mathrm{~J}=8 \mathrm{~Hz}$ ), 2.57 (t, $\left.{ }^{3} \mathrm{~J}=8 \mathrm{~Hz}, 4 \mathrm{H},-\mathrm{CH}_{2}-\mathrm{CH}_{2}-\mathrm{CN}\right), 2.07\left(\mathrm{t},{ }^{3} \mathrm{~J}=8 \mathrm{~Hz}, 4 \mathrm{H},-\mathrm{CH}_{2}-\mathrm{CH}_{2}-\mathrm{CN}\right.$ ) $), 1.96(\mathrm{t}$, $\left.{ }^{3} \mathrm{~J}=8 \mathrm{~Hz}, 2 \mathrm{H},-\mathrm{C}_{2}-\mathrm{C}_{\text {quart }}\right), 1.73(\mathrm{t}, 1 \mathrm{H},-\mathrm{S} \underline{\mathrm{H}}) .{ }^{13} \mathrm{C} \mathrm{NMR}\left(100 \mathrm{MHz}, \mathrm{CDCl}_{3}\right)$ $\delta$, ppm: $119.96\left(\mathrm{C}_{\text {quart }}-\underline{C} \mathrm{~N}\right), 117.89\left(-\mathrm{CH}_{2}-\underline{\mathrm{CN}}\right), 40.62\left(\underline{\mathrm{C}}_{\text {quart }}\right), 36.65$ ($\left.\underline{\mathrm{C}}_{2}-\mathrm{SH}\right) 35.78\left(\mathrm{~S}-\mathrm{CH}_{2}-\underline{\mathrm{CH}}_{2}-\mathrm{C}_{\text {quart }}-\right), 31.63\left(\underline{\mathrm{C}}_{2}-\mathrm{CH}_{2}-\mathrm{CN}\right), 26.79\left(\mathrm{~S}-\underline{\mathrm{CH}}_{2}-\right.$ $\left.\left.\mathrm{CH}_{2}-\mathrm{C}_{\text {quart }}\right)^{-}\right), 24.89\left(\mathrm{HS}-\mathrm{CH}_{2}-\underline{C}_{2}-\mathrm{S}\right), 13.48\left(\underline{C H}_{2}-\mathrm{CN}\right) . \mathrm{MS}(\mathrm{El}+) \mathrm{m} / \mathrm{z}(\%)$ : 61.01 (100), 69.05 (15.55), 207.08 (17.18), 267.08 (13.02). EA: calculated: [C] 53.90\% [H] 6.41\% [N] 15.71\% [S] 23.98 found: [C] $53.78 \%[\mathrm{H}] 6.60 \%[\mathrm{~N}] 15.43 \%[\mathrm{~S}] 23.82 \%$.

Compounds16\&17: To a solution of allyl malononitrile (53 g, $0.5 \mathrm{~mol})$ dissolved in freshly distilled THF $(500 \mathrm{ml}), 1,2-$ ethanedithiol $(235 \mathrm{ml}, 2.5 \mathrm{~mol}$ ) and DMPA ( $2.5 \mathrm{~g}, 0.002 \mathrm{~mol})$ was added under argon atmosphere. The reaction mixture was irradiated for 5 minutes. All volatiles were removed at $80{ }^{\circ} \mathrm{C}$ in 
vacuo (10 mbar). The residue was purified by $\mathrm{SiO}_{2}$ column chromatography (heptane/ ethyl acetate $\rightarrow$ methanol $\rightarrow$ DMF) to give 17 ( $2 \mathrm{~g}, 2 \%$ ) as colourless crystals and 16 (40 g, 40\%) as pale brownish, viscous liquid.

16: ${ }^{1} \mathrm{H}$ NMR (400 MHz, N,N-dimethylformamide-d $\mathrm{d}_{7}$ ) $\delta$, ppm: $3.83\left(\mathrm{t},{ }^{3} \mathrm{~J}=6.8 \mathrm{~Hz}, 1 \mathrm{H},(\mathrm{CN}) \mathrm{CH}_{-}-\mathrm{CH}_{2}-\right), 2.79-2.69(\mathrm{~m}, 4 \mathrm{H},-\mathrm{S}-$ $\left.\left(\mathrm{C}_{2}\right)_{2}-\mathrm{SH}\right), 2.64\left(\mathrm{t},{ }^{3} \mathrm{~J}=7.1 \mathrm{~Hz}, 2 \mathrm{H}, \mathrm{CH}_{2}-\mathrm{CH}_{2}-\mathrm{S}-,\right), 2.21-2.15(\mathrm{~m}$, $\left.2 \mathrm{H}, \mathrm{CH}-\mathrm{CH}_{2}-\mathrm{CH}_{2}\right), 1.96-1.88\left(\mathrm{~m}, 2 \mathrm{H}, \mathrm{CH}_{2}-\mathrm{C}_{2}-\mathrm{CH}_{2}\right), 1.72$ (t, $\left.{ }^{3} J=8.0 \mathrm{~Hz}, 1 \mathrm{H}, \mathrm{CH}_{2}-\mathrm{S}-\underline{H},\right) .{ }^{13} \mathrm{C} \mathrm{NMR}\left(100 \mathrm{MHz} \mathrm{CDCl}_{3}\right) \delta$, ppm: $112.47\left((\underline{\mathrm{CN}})_{2}-\mathrm{CH}-\right), 36.11\left(-\underline{\mathrm{C}} \mathrm{H}_{2}-\mathrm{CH}_{2}-\mathrm{SH}\right), 30.62\left(-\underline{\mathrm{CH}}_{2}-\mathrm{CH}_{2}-\mathrm{S}-\right)$, $29.64\left(\mathrm{CH}-\mathrm{CH}_{2}-\mathrm{CH}_{2}\right), 26.11\left(\mathrm{CH}_{2}-\underline{C H}_{2}-\mathrm{CH}_{2}\right), 24.72\left(\mathrm{CH}_{2}-\underline{\mathrm{CH}_{2}}-\mathrm{SH}\right)$, $22.48\left((\mathrm{CN})_{2}-\underline{\mathrm{CH}}-\right)$.

17: ${ }^{1} \mathrm{H}$ NMR (400 MHz, $\mathrm{N}, \mathrm{N}$-dimethylformamide-d $\left.\mathrm{d}_{7}\right) \delta$, ppm: 6.63 (br s, $-\mathrm{N}_{2}$ ), 6.37 (br s, $-\mathrm{N}_{\mathrm{H}_{2}}$ ), 3.20-3.30 (m, $2 \mathrm{H},-\mathrm{CH}_{2}-\mathrm{CH}_{2}-$ $\mathrm{S}-\mathrm{C}), 2.74-2.79\left(\mathrm{~m}, 2 \mathrm{H}, \mathrm{CH}_{2}-\mathrm{S}-\mathrm{C}_{2}-\mathrm{CH}_{2}\right), 2.61-2.68\left(\mathrm{~m}, 2 \mathrm{H}, \mathrm{CH}_{2}-\right.$ $\left.\mathrm{C}_{2}-\mathrm{CH}_{2}-\mathrm{S}\right), 1.69-1.81\left(\mathrm{~m}, 2 \mathrm{H}, \mathrm{CH}_{2}-\mathrm{C}_{2}-\mathrm{CH}_{2}\right), 2.27-2.40(\mathrm{~m}, 2 \mathrm{H}$, $\left.\mathrm{C}-\mathrm{C}_{2}-\mathrm{CH}_{2}\right) .{ }^{13} \mathrm{C}$ NMR (100 MHz, N,N-dimethyformamide-d $\left.\mathrm{d}_{7}\right) \delta$, ppm: $152.17\left(\left(\mathrm{C}=\underline{C}\left(\mathrm{NH}_{2}\right)-\mathrm{S}\right), 122.13(\mathrm{C}-\underline{\mathrm{CN}}), 80.72(\mathrm{C}=\underline{\mathrm{C}}(\mathrm{CN})-)\right.$, $31.97\left(\mathrm{CH}_{2}-\underline{\mathrm{C}} \mathrm{H}_{2}-\mathrm{S}-\mathrm{C}\right), 31.21\left(\underline{\mathrm{C}} \mathrm{H}_{2}-\mathrm{CH}_{2}-\mathrm{S}-\mathrm{C}\right), 30.53\left(\mathrm{CH}_{2}-\mathrm{CH}_{2}-\underline{\mathrm{C}} \mathrm{H}_{2}-\right.$ S), $28.55\left(\mathrm{CH}_{2}-\mathrm{CH}_{2}-\mathrm{CH}_{2}-\mathrm{S}\right), 27.16\left(\mathrm{CH}_{2}-\mathrm{CH}_{2}-\mathrm{CH}_{2}-\mathrm{S}\right)$. HRMS (ESI): calc. for $\mathrm{C}_{16} \mathrm{H}_{25} \mathrm{~N}_{4} \mathrm{~S}_{4}[\mathrm{M}+\mathrm{H}]$ 401.0957; found: 401.0957 .

\section{Results and Discussion}

Allyl cyanide is the simplest aliphatic non-conjugated vinyl nitrile. The thiol-ene addition of $\mathbf{A}$ to allyl cyanide initiated by $1 \mathrm{~mol} \%$ DMPA gave S-3-cyanopropyl thioacetate 1 in 73\% isolated yield within 5 min (Scheme 1). Compound $\mathbf{1}$ is known in the literature and was prepared starting from potassium salt of $\mathbf{A}$ and 4bromobutyronitrile. Unfortunately, no reaction yield is given for this reaction. $^{34,35}$ Cleavage of the acetyl moiety of $\mathbf{1}$ under acidic conditions (20 mol\% trimethylsilyl chloride (TMSCl) in methanol) proceded at low reaction rate and gave 4-mercaptobutanenitrile 2 in moderate yields (43\%). When a stoichiometric amount of $\mathrm{TMSCl}$ was used, 2-iminothiolane hydrochloride 3 formed in $60 \%$ yield. Compound $\mathbf{2}$ is known and was prepared starting from 4isothioureidobutyronitrile hydrochloride but the reaction yield was very low (15\%). ${ }^{36}$ Compound $\mathbf{3}$ is also known in the literature and was prepared by nucleophilic substitution starting from 4halogenobutanenitrile and $\mathbf{A}$ or thiourea, respectively. ${ }^{37-39}$ It is widely used as thiolation reagent in biochemistry and is referred to as 'Traut's reagent'. ${ }^{40-43}$

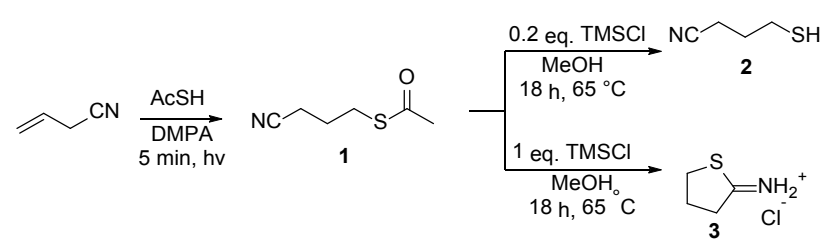

Scheme 1 Thiol-ene addition of $\mathbf{A}$ to allyl cyanide followed by the cleavage of the acetyl group to give $\mathbf{2}$ and $\mathbf{3}$.

Allyl malononitrile $\mathbf{5}$ was synthesized according to the literature starting from malononitrile and allyl bromide in one step (Scheme 2). ${ }^{41}$ The thiol-ene addition of a slight excess of $\mathbf{A}$ to $\mathbf{5}$ in presence of
DMPA gave S-(4,4-dicyanobutyl) thioacetate 6 in 74\% yield within 5 $\min$ (Scheme 2). When a large excess of $\boldsymbol{A}$ was used, addition to the nitrile groups was observed. Acetate cleavage under basic conditions in presence of butyl amine resulted in quantitative cyclization of 6 to 6-amino-3,4-dihydro-2H-thiopyran-5-carbonitrile 8. The quantitative conversion of $\mathbf{6}$ to $\mathbf{8}$ is supported by the ${ }^{1} \mathrm{H}$ NMR spectra where the characteristic signals of 6 disappeared and new signals appeared which can be easily assigned to 8 (Figure 1 ). Single crystals of $\mathbf{8}$ suitable for $\mathrm{x}$-ray analysis were grown from ethyl acetate/heptane. The proposed six-membered ring was confirmed by $x$-ray structure (Figure 2). The transesterification reaction with methanol under acidic conditions allowed formation of 4,4dicyanobutyl thiol 7, but the reaction did not proceed to completion. Attempts to purify this compound by vacuum distillation were only partially successful. The compound still contained about $15 \%$ impurities (Figure 1d). Further attempts to increase the purity of $\mathbf{7}$ by column chromatography purification on silica gel were unsuccessful. The intramolecular cyclization of $\mathbf{7}$ occurred quantitatively with formation of $\mathbf{8}$ (Figure 1). The synthesis of the analog five-membered cycle starting from malononitrile and ethylene sulfide was already described in the literature, but an over-stoichiometric amount of a strong base such as sodium hydride had to be used and the reaction yield was only moderate $(57 \%){ }^{45}$

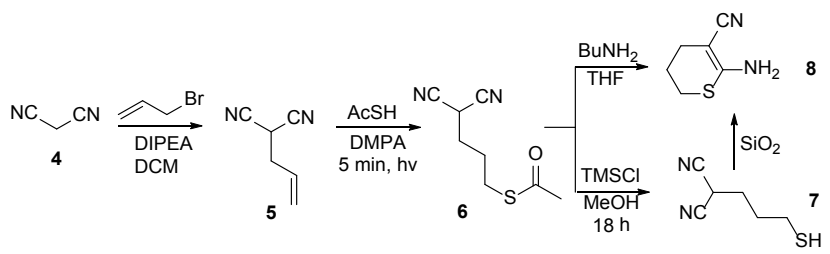

Scheme 2 Synthetic route to 4,4-dicyanobutyl thiol 7.

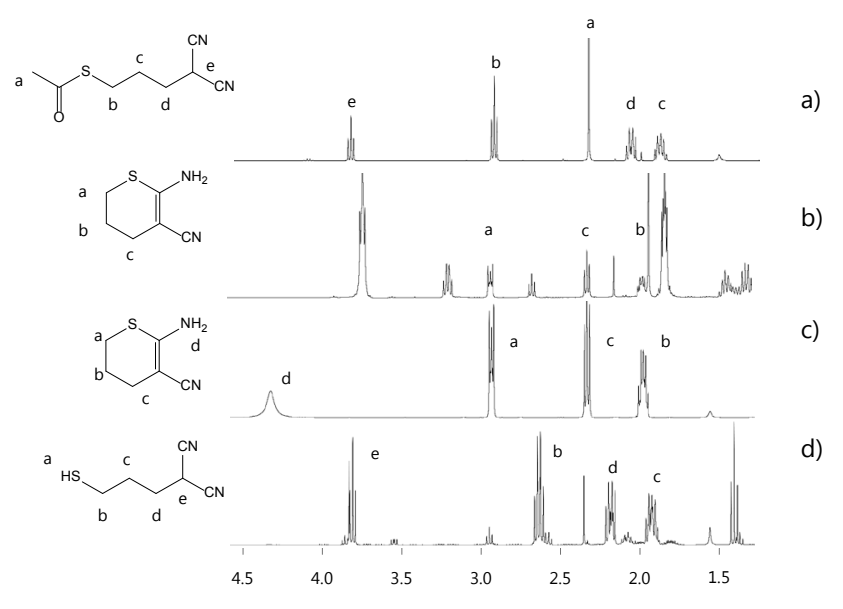

Fig. ${ }^{1} \mathrm{H}$ NMR spectra of: a) 6 , b) of the crude reaction mixture obtained by reacting 6 with butyl amine, c) of 8, and d) of $\mathbf{7}$. 

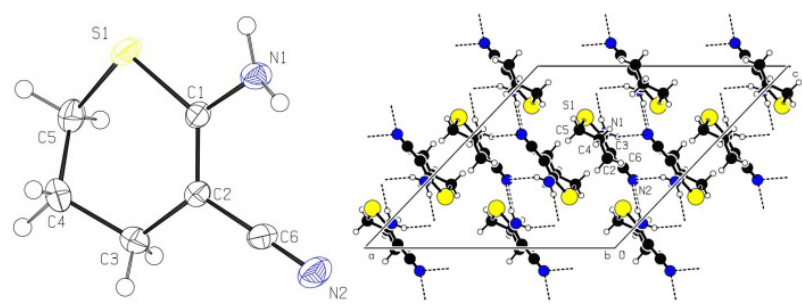

Fig. 2 ORTEP plot of 8 , view perpendicular to the cycle (left) and the molecular packing down the crystallographic $b$ axis showing the stacking of the cycles and the hydrogen bonding network (right).

3-vinylpentane-1,3,5-trinitrile 9 was synthesized according to literature starting from allyl cyanide (Scheme 3$).{ }^{46}$ The vinyl group of 9 is sterically hindered due to the bulky $-\left(\mathrm{CH}_{2}\right)_{2} \mathrm{CN}$ moieties in alpha position to the vinyl group. This is nicely illustrated by the ${ }^{1} \mathrm{H}$ NMR spectrum of 9 where the two protons of the $-\mathrm{CH}_{2}-\mathrm{CH}_{2}-\mathrm{CN}$ group next to the quaternary carbon are not equivalent, as one would expect (see ESI). This indicates a lack of free rotation of the $\left(\mathrm{CH}_{2}\right)_{2} \mathrm{CN}$ moiety. The thiol-ene addition of $\mathbf{A}$ to 9 initiated by DMPA did not occur even after 60 min UV irradiation. Instead, instability of the nitriles was observed. When AIBN as thermal radical initiator and excess of $\mathbf{A}$ was used, the addition of $\mathbf{A}$ to the nitrile group was observed. NMR analysis of the crude product mixture showed the formation of $\mathbf{1 1}$ which hydrolysed to 4-cyano-4-vinylheptane bis(thioamide) 12 during work-up (60\% yield). Formation of 3-(2thioacetic)ethylpentane-1,3,5-carbonitrile $\mathbf{1 0}$ was not observed. The conversion of aliphatic nitriles to thioamides is known, however this occurrs either under acidic or basic catalysis. ${ }^{30,31}$ At $70{ }^{\circ} \mathrm{C}$ the acidity of $\mathbf{A}$ was sufficient to catalyse its addition to the nitrile of $\mathbf{9}$.

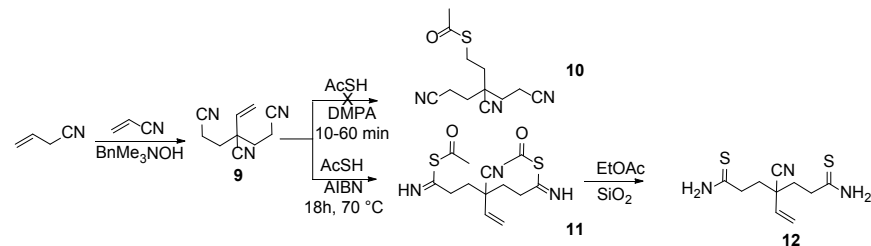

Scheme $\mathbf{3}$ Synthesis of $\mathbf{9}$ by cyanoethylation of allyl cyanide and its reaction with $\mathrm{AcSH}$.

As an alternative to $\mathbf{A}$ in the transformation of vinyl nitriles to mercaptonitriles, 1,2-ethanedithiol B was tested (Scheme 4). The advantage of $\mathbf{B}$ is that it allows formation of mercaptonitriles directly via thiol-ene reaction of vinyl nitriles, even though an excess of $\mathbf{B}$ has to be used and the spacer between the thiol and the nitrile group is prolonged. Intramolecular cyclization reactions of the formed mercaptonitriles are less favored as five or sixmembered rings cannot form

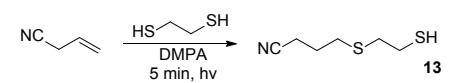

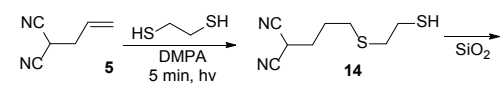

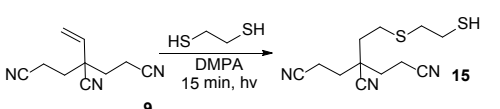

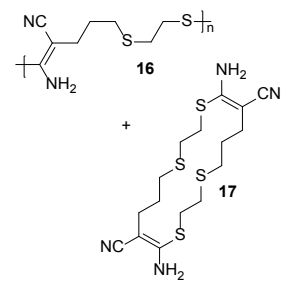

Scheme 4 Thiol-ene addition of 1,2-ethanedithiol to vinyl nitriles.

Addition of $\mathbf{B}$ to allyl cyanide gave 4-((2mercaptoethyl)thio)butanenitrile 13, which did not show any susceptibility to cyclization. The purification of $\mathbf{1 3}$ was done by distillation and the compound could be easily prepared on $30 \mathrm{~g}$ scale. The reaction of 5 with $\mathbf{B}$ gave 2-(3-)( 2 mercaptoethyl)thio)propyl)malononitrile 14 . The isolation of 14 by distillation was possible, but challenging given 14 's high boiling point. Again, silica gel during chromatography purification catalyzed the intramolecular cyclization with formation of cyclic dimer 17 in small amounts. The structure of $\mathbf{1 7}$ was confirmed by x-ray diffraction analysis on a single crystal (Figure 3 ). The main product after chromatography was oligomer 16 which was obtained via silica gel extraction with DMF and methanol as can be seen in the ${ }^{1} \mathrm{H}$ NMR spectrum in the supporting information. No further characterizations were conducted on $\mathbf{1 6}$ due to its poor solubility.
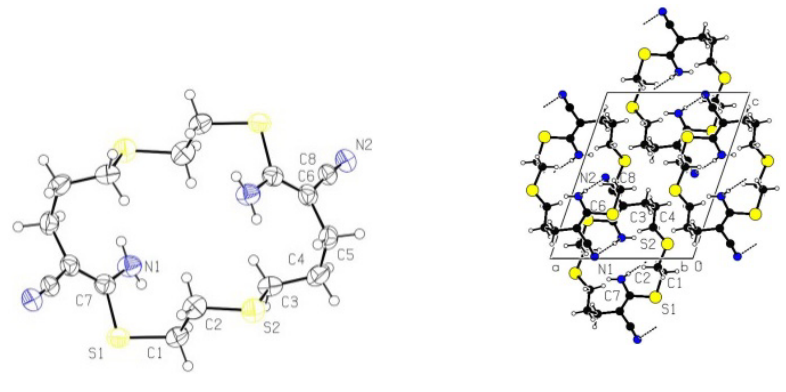

Fig. 3 ORTEP plot of 17, view perpendicular to the cycle (left) and the molecular packing down the crystallographic a axis showing the hydrogen bonding network (right).

In contrast to $\mathbf{A}$, selective thiol-ene addition of $\mathbf{B}$ to the vinyl group of $\mathbf{9}$ was achieved by using $\mathbf{B}$ in excess, an over-stoichiometric amount of photoinitiator, and prolonged reaction times. The difference in the reactivity of $\mathbf{A}$ and $\mathbf{B}$ can be explained by the acidity of the two reagents used. ${ }^{47}$ Due to the presence of electronwithdrawing group, $\mathbf{A}$ is more acidic as compared to 1,2ethanedithiol and therefore chemically more reactive towards nitrile. NMR analysis of the reaction mixture showed product conversion of approximately 50\%. The nitrile groups were unaffected during reaction and chromatography purification process. The mercaptonitriles 13-15 did not crystallize. Traces of Markovnikov addition product were detected in all cases.

Thiols $\mathbf{1 3}$ and $\mathbf{1 4}$ were identified as being promising candidates for the post polymerization modification of polyvinylsiloxanes via thiol-ene addition. 13 and $\mathbf{1 4}$ can be prepared easily on a $20 \mathrm{~g}$ scale and can be purified by distillation. Although the high boiling points 
of these thiols may be considered disadvantageous since high vacuum and high temperatures have to be used for their distillation, they have the advantage that they are less volatile and therefore do not have the typical pungent odor characteristic for thiols. The thiols show a dielectric permittivity at high frequencies of $\varepsilon^{\prime}=15.4$ and $\varepsilon^{\prime}=17$ for 13 and $\mathbf{1 4}$, respectively. Preliminary results show that $\mathbf{1 3}$ and $\mathbf{1 4}$ can be successfully used in the selective thiol-ene addition to polyvinylsiloxane and no side reactions were observed. Further work in our lab is conducted in this direction.

\section{Conclusions}

In conclusion, the thiol-ene addition of $\mathbf{A}$ and $\mathbf{B}$ to allyl cyanide, allyl malononitrile, and 3-vinylpentane-1,3,5-carbonitrile was investigated. All vinyl nitriles reacted with $\mathbf{A}$ and $\mathbf{B}$ as expected except 3-vinylpentane-1,3,5-carbonitrile. This compound with its sterically hindered vinyl group did not give the thiol-ene addition product $\mathbf{A}$ but the addition product to the nitriles. The cleavage of the acetyl groups to generate the respective mercaptonitriles must be conducted under mild acidic conditions due to the high tendency of the generated thiols to undergo addition to the nitriles under acidic and basic conditions. Purification of the mercaptonitriles by column chromatography over silica gel should be avoided. Thiol-ene addition of $\mathbf{B}$ to non-conjugated vinyl nitrile compounds proved to be a fast and efficient alternative for $\mathbf{A}$ in the synthesis of mercaptonitriles in one step and mild reaction conditions.

\section{Acknowledgements}

We gratefully acknowledge Swiss Federal Laboratories for Materials Science and Technology (Empa, Dübendorf) for financial support.

\section{Notes and references}

1 V. N. Ipatieff and B. S. Friedman, J. Am. Chem. Soc., 1939, 61, 71.

2 C. E. Hoyle and C. N. Bowman, Angew. Chem. Int. Ed., 2010, 49, 1540.

3 L. Gu, Q. Xue, S. Peng, G. Wang, J. Han and X. Wu, Polymer, 2016, 7, 625.

4 C. Ligeour, L. Dupin, A. Marra, G. Vergoten, A. Meyer, Y. Dondoni, A. Souteyrand, E. Vasseur, J.-J. Chevolot and F. Morvan, Eur. J. Org. Chem., 2014, 34, 7621.

5 Z. Liu, T. Liu, Q. Lin, C. Bao and L. Zhu, Angew. Chem. Int. Ed., 2015, 54, 174.

6 Y. Suzuki, T. Higashihara, S. Ando and M. Ueda, Macromolecules, 2012, 45, 3402.

7 N. K. Singha, M. I. Gibson, B. P. Koiry, M. Danial and H.-A. Klok, Biomacromolecules, 2011, 12, 2908.

8 M. H. Stenzel, ACS Macro Lett., 2013, 2, 14.

9 Y. Chen, G. Chen and M. H. Stenzel, Macromolecules, 2010, 43, 8109.

10 J. Z. Du, X. J. Du, C. Q. Mao and J. Wang, J. Am. Chem. Soc., 2011, 133, 17560.

11 M. Podgorski, E. Becka, M. Claudino, A. Flores, P. K. Shah, J. W. Stansbury and C. N. Bowman, Dent. Mater., 2015, 31, 1255.

12 E. Klemm and S. Sensfuss, Makromol. Chem., 1991, 64,
159.

13 A. S. Quick, J. Fischer, B. Richter, T. Pauloehrl, V. Trouillet, M. Wegener and C. Barner-Kowollik, Macromol. Rapid Commun., 2013, 34, 335.

14 C. S. Marvel and A. Kotch, J. Am. Chem. Soc., 1951, 73, 1100.

15 S. J. Duenki, Y. S. Ko, F. A. Nueesch and D. M. Opris, Adv. Funct. Mater., 2015, 25, 2467.

16 S. J. Dünki, M. Tress, F. Kremer, S. Y. Ko, F. A. Nüesch, C.D. Varganici, C. Racles and D. M. Opris, RSC Adv., 2015, 5, 50054.

17 C. Decker and T. Nguyen Thi Viet, Polymer, 2000, 41, 3905.

18 A. Gress, A. Vo and H. Schlaad, Macromolecules, 2007, 40, 7928.

19 J. C. Persson, K. Josefsson and P. Jannasch, Polymer, 2006, 47, 991.

20 M. Li, P. De, H. Li and B. S. Sumerlin, Polym. Chem., 2010, $1,854$.

21 D. M. Opris, E. Perju and S. J. Duenki, J. Polym. Sci. Part A: Polym. Chem., 2016, 56, 2940.

22 M. Le Neindre, B. Magny and R. Nicolaÿ, Polym. Chem., 2013, 4, 5577.

23 J. Polster and P. Schieberle, J. Agric. Food Chem., 2015, 63, 1419.

24 E. Schjanberg, Ber. Dtsch. Chem. Ges., 1941, 74, 1751.

25 R. Brown, W. E. Jones and A. R. Pinder, J. Chem. Soc, 1951, 2123.

26 J. Gorges and U. Kazmaier, Eur. J. Org. Chem., 2015, 36, 8011.

27 J. W. Lynn, R. L. Roberts and J. R. Kilsheimer, J. Org. Chem., 1961, 26, 4300.

28 A. Kjaer and J. Conti, Acta Chem. Scand., 1954, 8, 295.

29 M. Yunhai and X. Jiaxi, Synthesis, 2012, 44, 2225.

30 J. Y. Gauthier and H. Lebel, Phosphorus, Sulfur Silicon Relat. Elem., 1994, 95, 325.

31 K. A. Mahammed, V. P. Jayashankara, N. Rai, K. Raju, K. Mohana and P. N. Arunachalam, Synlett, 2009, 14, 2338.

32 R. Jue, J. M. Lambert, L. R. Pierce and R. R. Traut, Biochemistry, 1978, 17, 5399.

33 G. R. Fulmer, A. J. M. Miller, N. H. Sherden, H. E. Gottlieb, A. Nudelman, B. M. Stoltz, J. E. Bercaw and K. I. Goldberg, Organometallics, 2010, 29, 2176.

34 N. Park, K. Park, M. Jang and S. Lee, J. Org. Chem., 2011, 76, 4371-4378.

35 Los, M. (American Cyanamid Co.) Eur. Pat. Appl. EP 133310, 1985.

36 R. R. Traut, A. Bollen, T. T. Sun, J. W. B. Hershey, J. Sundberg and L. R. Pierce, Biochemistry, 1973, 12, 3266.

37 M. Wallace, A. J. Allentoff, J. Brailsford, S. Gong, S. Bonacorsi and F. Rinaldi, J. Label. Compd. Radiopharm., 2015, 58, 429.

38 S. F. Carroll and D. A. Goff, 1990, WO 90/06774.

39 T. P. King, Y. Li and L. Kochoumian, Biochemistry, 1978, 17, 1499.

40 T. P. King, Y. Li and L. Kochoumian, Biochemistry, 1986, 25, 5774.

41 A. Braden, M. Roner, J. Ganter and K. Nelson, J. Nanosci. 
Nanotechnol., 2007, 7, 925.

42 T. Tada, K. Mano, E. Yoshide, N. Tanaka and S. Kunugi, Bull. Chem. Soc. Jpn., 2002, 75, 2247.

43 T. T. Ngo, Appl. Biochem. Biotechnol., 1986, 13, 213.

44 M. K. Ghorai, R. Talukdar and D. P. Tiwari, Org. Lett., 2014, 16, 2204.

45 K. Yamagata, T. Yukihiko, M. Yamazaki, T. Matsuda and K. Noda, Chem. Pharm. Bull., 1982, 30, 4396.

46 H. A. Bruson and T. W. Riener, J. Am. Chem. Soc., 1943, 65, 18.

47 B. Dmuchovsky, F. B. Zienty and W. A. Vredenbur, J. Org. Chem., 1966, 31, 865. 